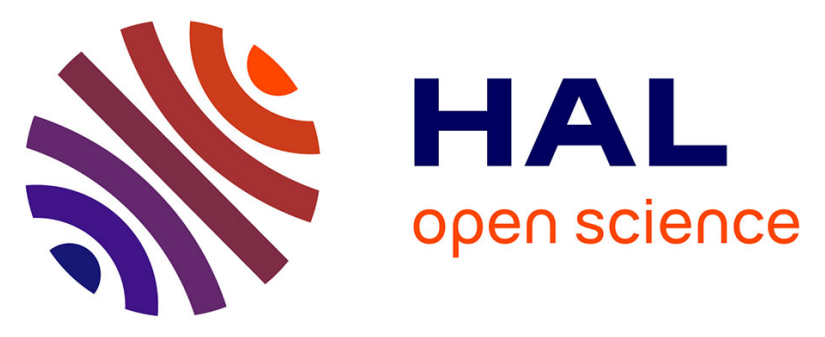

\title{
Synthesis of platinum complexes of Fluorenyl-substituted Porphyrins used as Phosphorescent dyes for solution-processed organic light-emitting devices.
}

Christine Paul-Roth, Samuel Drouet, Areej Merhi, J. A. Gareth Williams, Louise F. Gildea, Christopher Pearson, Michael C. Petty

\section{To cite this version:}

Christine Paul-Roth, Samuel Drouet, Areej Merhi, J. A. Gareth Williams, Louise F. Gildea, et al.. Synthesis of platinum complexes of Fluorenyl-substituted Porphyrins used as Phosphorescent dyes for solution-processed organic light-emitting devices.. Tetrahedron, 2013, 69 (46), pp.9625-9632. 10.1016/j.tet.2013.09.034 . hal-00914491

\author{
HAL Id: hal-00914491 \\ https://hal.science/hal-00914491
}

Submitted on 5 Dec 2013

HAL is a multi-disciplinary open access archive for the deposit and dissemination of scientific research documents, whether they are published or not. The documents may come from teaching and research institutions in France or abroad, or from public or private research centers.
L'archive ouverte pluridisciplinaire HAL, est destinée au dépôt et à la diffusion de documents scientifiques de niveau recherche, publiés ou non, émanant des établissements d'enseignement et de recherche français ou étrangers, des laboratoires publics ou privés. 
Complete Author Information for Dr Christine O. Paul-Roth

\section{Affiliation :}

INSA-ISCR - Université de Rennes I

UMR-6226

Campus de Beaulieu

35042 Rennes Cedex - France

e-mail adress: christine.paul@univ-rennes1.fr and christine.paul@insa-rennes.fr

Tél : (33) 0223236372

Fax : (33) 0223235637 


\section{Graphical Abstract}

\section{Synthesis of platinum complexes of Fluorenyl-substituted Porphyrins used as}

Phosphorescent dyes for solution-processed organic light-emitting devices

Christine O. Paul-Roth, ${ }^{* a, b}$ Samuel Drouet, ${ }^{a}$ Areej Merhi, ${ }^{b}$ J. A. Gareth Williams, ${ }^{* c}$ Louise F. Gildea, ${ }^{c}$ Christopher Pearson $^{* d}$ and Michael C. Petty ${ }^{d}$

${ }^{a}$ Institut des Sciences Chimiques de Rennes, ISCR-UMR CNRS 6226, Université de Rennes I, 35042 Rennes Cedex, France.

${ }^{b}$ Institut National des Sciences Appliquées de Rennes, INSA-ISCR, 20 Avenue Buttes de Coësme, 35043 Rennes, France.

${ }^{c}$ Department of Chemistry, Durham University, Durham, DH1 3LE, U.K.

${ }^{d}$ School of Engineering and Centre for Molecular and Nanoscale Electronics, Durham University, Durham, DH1 3LE, U.K.

Synthesis and photophysical properties of new platinum(II) complexes of porphyrins with four (PtTOFP) or eight (PtOOFP) fluorenyl pendant arms have been studied. OLED's in which these platinum(II) complexes are used as a red phosphor have been prepared.
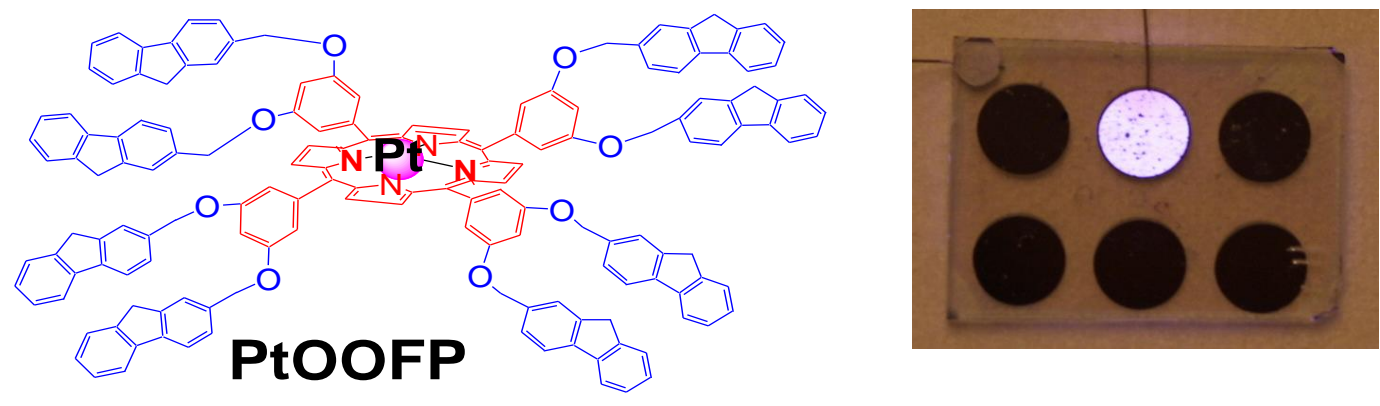


\title{
Synthesis of platinum complexes of Fluorenyl-substituted Porphyrins used as Phosphorescent dyes for solution-processed organic light-emitting devices
}

\author{
Christine O. Paul-Roth, ${ }^{a, b}$ Samuel Drouet, ${ }^{a}$ Areej Merhi, ${ }^{b}$ J. A. Gareth Williams, ${ }^{c}$ \\ Louise F. Gildea, ${ }^{c}$ Christopher Pearson ${ }^{d}{ }^{d}$ and Michael C. Petty ${ }^{d}$ \\ ${ }^{a}$ Institut des Sciences Chimiques de Rennes, ISCR-UMR CNRS 6226, \\ Université de Rennes I, 35042 Rennes Cedex, France. \\ ${ }^{b}$ Institut National des Sciences Appliquées de Rennes, INSA-ISCR \\ 20 Avenue Buttes de Coësme, 35043 Rennes, France. \\ ${ }^{c}$ Department of Chemistry, Durham University, Durham, DH1 3LE, U.K. \\ ${ }^{d}$ School of Engineering and Centre for Molecular and Nanoscale Electronics, \\ Durham University, Durham, DH1 3LE, U.K.
}

Platinum(II) complexes of symmetrical meso-substituted $\mathrm{A}_{4}$-porphyrins bearing four fluorene donor moieties 5,10,15,20-tetra(4-(2 methyloxyfluorenyl)phenyl)porphyrin, referred to as TOFP, or eight fluorene arms, 5,10,15,20-octa(3,5-(2 methyloxyfluorenyl)phenyl)porphyrin, OOFP, were synthesised and characterised. The photophysical properties of the new compounds are reported and compared to those of PtTPP and PtTFP $\{$ TPP = tetraphenylporphyrin and TFP = tetra(2-fluorenyl)porphyrin $\}$. The luminescence quantum yields of PtTOFP and PtOOFP are $11 \%$ and $4.2 \%$ respectively compared to $4.6 \%$ for the reference PtTPP and only $2.0 \%$ for the previously studied compound PtTFP. The electronic and optoelectronic behaviour of solutionprocessed organic light-emitting devices (OLEDs) are reported that incorporate these platinum porphyrins as phosphorescent dyes doped into different layers of a polyvinylcarbazole (PVK) host.

Keywords: porphyrins; platinum; fluorene; phosphorescence; light harvesting; quantum yield; OLEDs.

\section{Introduction}

Organic light-emitting diodes (OLEDs) are of intense interest in the development of flat-panel display devices and for energy-efficient lighting. ${ }^{1,2}$ In both cases, three primary colours, blue, green, and red, are necessary. For commercial applications, the most success has been obtained for green emitters. ${ }^{3}$ For the blue light-emitting materials, such as polyfluorenes, high efficiency has been achieved, but there are frequent problems with stabilities and lifetimes. ${ }^{4}$ The development of red emitting systems is also challenging, partly due to the drop-off in emission quantum yield that is typically observed for lower energy excited states. This trend stems from 
the fact that non-radiative deactivation of excited states through intramolecular energy transfer into vibrations is favoured as the energy decreases (often referred to as the "energy gap law") and also from the dependence of the radiative rate constant on $v^{3}$, according to the Einstein coefficient for spontaneous emission ( $v=$ frequency of light emitted).

Many porphyrins emit quite strongly in the red region of the spectrum, owing to their rigid, highly conjugated structures. Moreover, they have quite narrow bandwidths, potentially favouring high colour purity if used in an OLED. Some studies have indeed used porphyrins as red OLED emitters; for example, a device comprising what is probably the most accessible of the porphyrin family, namely tetraphenylporphyrin (TPP), doped into a conjugated polymer, poly(9,9-dioctylfluorene), was described several years ago. ${ }^{5}$ The importance of fluorene units in organic electronics and photonics makes the combination of porphyrins and fluorenes particularly intriguing. For example, a series of star-shaped porphyrins bearing pendent oligofluorene arms were reported by Bo and co-workers. ${ }^{6,7}$ In previous work from our laboratory, we reported the synthesis of 5,10,15,20-tetrafluorenylporphyrin (TFP), incorporating fluorenyl substituents at the four meso positions of the porphyrin macrocycle, together with the catalytic properties of its ruthenium(II) complex. ${ }^{8-13}$ In terms of the photophysical properties, the fluorenyl substituents were found to lead to an increase in the fluorescence quantum yield of the free-base porphyrin $\left(\Phi_{\mathrm{F}}=0.24\right)$ relative to that of $\operatorname{TPP}\left(\Phi_{\mathrm{F}}=0.13\right.$ under the same conditions $)$, apparently owing largely to an increase in the radiative rate constant associated with distortion of the porphyrin macrocycle. ${ }^{14}$ A density functional theory study on such compounds has been carried out by others. ${ }^{15}$ We have also prepared dendrimeric supramolecular assemblies bearing 12 and 24 fluorenyl peripheral donor groups surrounding the porphyrin core. ${ }^{16-18}$

Over the past decade, many studies have focused on the use of luminescent organometallic complexes as emitting species in OLEDs. The high spin-orbit coupling constants associated with third-row transition metal ions such as Ir(III) and $\mathrm{Pt}(\mathrm{II})$ promotes the emission of light from triplet states that are formed upon charge recombination in an OLED in ratios as high as 3:1 over the singlets. In a device comprising a purely organic emitting material, the emission from the triplet states is forbidden through the spin selection rule $\Delta S=0$, limiting the maximum attainable internal efficiency to $25 \%$. Transition metal-based systems allow this limit to be raised to $100 \%$. Platinum(II) complexes of porphyrins are therefore of interest as OLED emitters. Indeed, the first tests on the use of phosphorescent transition metal complexes as OLED phosphors were carried out with platinum octaethylporphyrin (PtOEP). ${ }^{19}$

Recently, we prepared the Pt(II) complex of the tetrafluorenylporphyrin discussed above, PtTFP, and successfully incorporated it into a simple two-layer OLED device. ${ }^{20}$ In the present paper, we describe two new platinum complexes of porphyrins based around a TPP core carrying 
either four or eight fluorene arms, PtTOFP and PtOOFP respectively (Figure 1). In contrast to the PtTFP system, the fluorenyl units in these new compounds are not directly linked to, or conjugated with, the porphyrin ring. They are separated from the meso-phenyl rings via insulating $-\mathrm{OCH}_{2}-$ linkers. The photophysical properties of the new compounds are investigated in solution, and we also report on the use of these complexes as emitters in simple OLED devices prepared by spin-coating procedures.

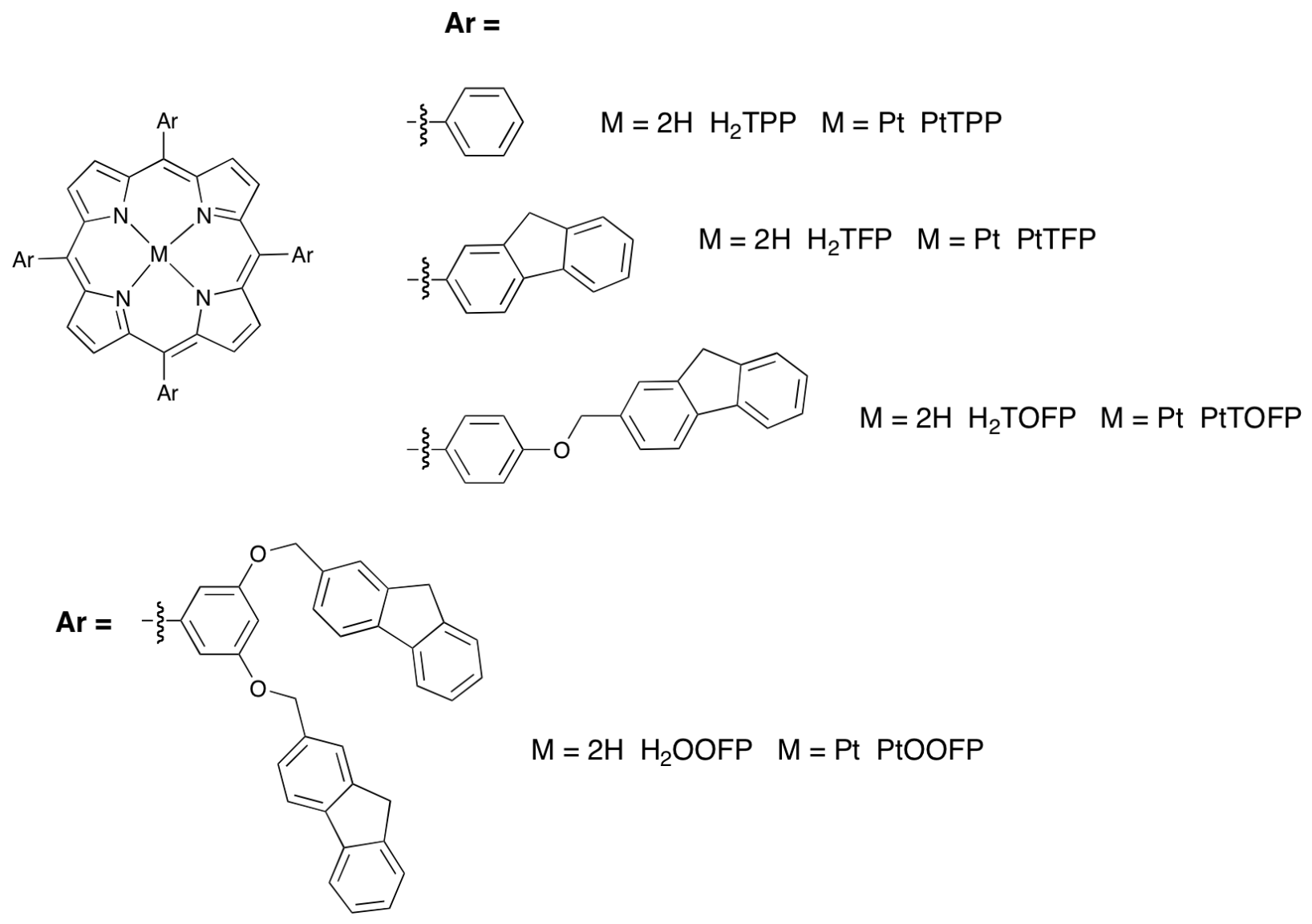

< Figure 1: Structures of the free-base porphyrins and their platinum(II) complexes >

\section{Results and discussion}

\section{Synthesis}

The two free-base porphyrins $\mathrm{H}_{2}$ TOFP and $\mathrm{H}_{2} \mathrm{OOFP}$ were synthesised as we reported previously. ${ }^{16}$ Their platinum(II) complexes were prepared by using a method adapted from that used for the formation of PtTPP. ${ }^{21}$ The platinum salt $\mathrm{PtCl}_{2}$ was dissolved in benzonitrile and pre-heated at $100{ }^{\circ} \mathrm{C}$ for $1 \mathrm{~h}$ under an argon atmosphere, followed by addition of the free-base porphyrin and reflux for a further $2 \mathrm{~h}$ (Scheme 1). The crude products were purified by chromatography on silica to give the desired compounds PtTOFP and PtOOFP as reddish-purple solids, which were characterised by NMR and UV-visible spectroscopy and mass spectrometry. It should be noted that the key difference between $\mathrm{H}_{2} \mathrm{OOFP}$ and $\mathrm{H}_{2}$ TOFP (and likewise between 
the two platinum complexes) is in the number of appended fluorenyl groups: eight versus four respectively. Although the fluorenyl groups are linked to the pendent aryl groups at different positions (meta versus para in $\mathrm{H}_{2} \mathrm{OOFP}$ and $\mathrm{H}_{2}$ TOFP respectively), this differing substition pattern is expected to have minimal effect on the porphyrin-based electronic excited state, given the expected twist of the pendent aryls groups relative to the porphyrinic plane. Indeed, we have previously found that the corresponding model 4-methoxyphenyl and 3,5-dimethoxyphenylsubstituted porphyrins differ by less than $5 \mathrm{~nm}$ in their fluorescence emission maxima.

\section{Absorption and emission spectra in solution}

(i) Free-base porphyrins. The UV-visible spectra of the free-base porphyrins exhibit a combination of bands as anticipated from the constituent units. Spectra for $\mathrm{H}_{2} \mathrm{TOFP}$ are shown in Figure 2, and data for both it and for $\mathrm{H}_{2} \mathrm{OOFP}$ are compiled in Table 1. The intense Soret bands around $420 \mathrm{~nm}$ and Q bands around 590 and $650 \mathrm{~nm}$, characteristic of meso-tetraarylporphyrins, are accompanied by bands around $270 \mathrm{~nm}$ associated with the fluorene substituents. In both cases, the Soret and $\mathrm{Q}$ bands are a little red-shifted compared to $\mathrm{H}_{2} \mathrm{TPP}$, more so for $\mathrm{H}_{2}$ TOFP, probably owing to the para-disposition of the electron-donating alkoxy substituent in this case.

Closer inspection of the absorption spectrum in Figure 2 reveals the presence of an unexpected additional band around $450 \mathrm{~nm}$, to low energy of the Soret, and a long-wavelength tail to the lowest-energy of the four $\mathrm{Q}$ bands. These features do not appear in the fluorescence excitation spectrum (vide infra), and are probably due to the presence of a trace amount of the protonated porphyrin (unstabilised $\mathrm{CH}_{2} \mathrm{Cl}_{2}$ typically contains trace $\mathrm{H}^{+}$). The free-base porphyrins have very low solubility, such that the more soluble protonated form may appear unexpectedly intense in the absorption spectrum of a saturated solution of the porphyrin. Support for this interpretation is provided by the fact that, upon addition of trifluoroacetic acid, there is a large shift in the Soret band to $450 \mathrm{~nm}$ and a broad featureless band appears at $700 \mathrm{~nm}$ (Figure 3). 


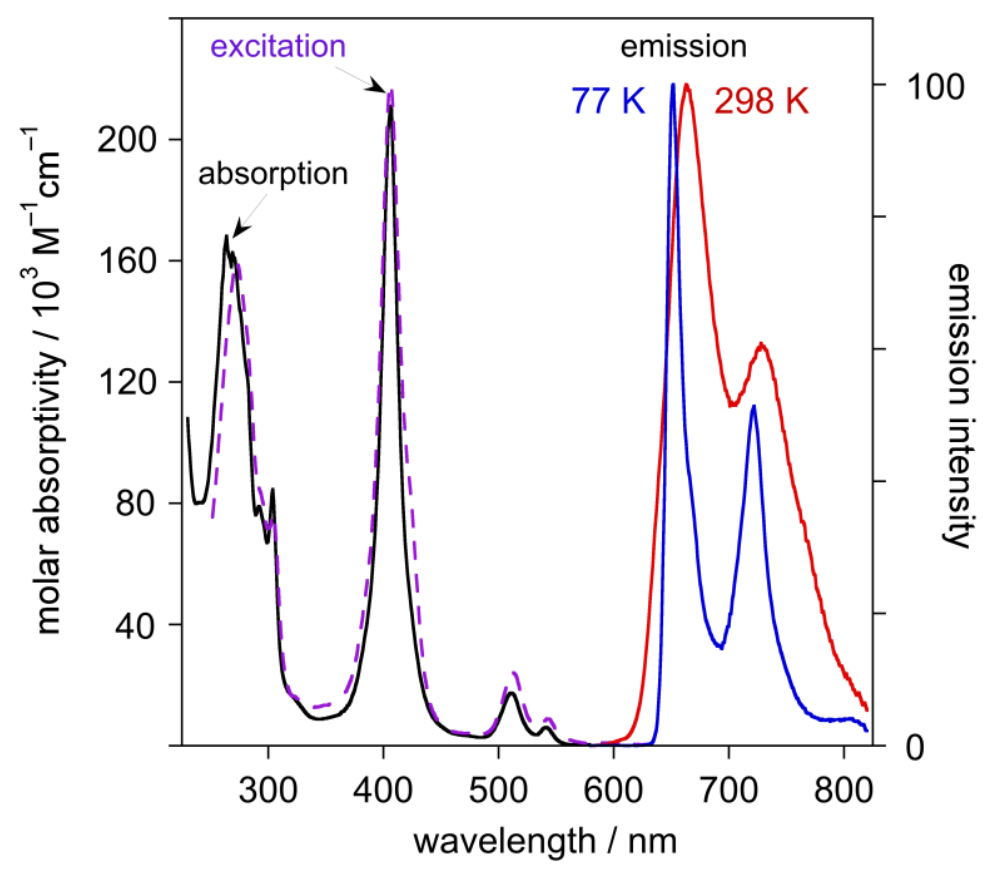

< Figure 2: UV-visible absorption spectrum (black line), emission spectrum (red line) and excitation spectrum ( $\lambda_{\mathrm{em}}=725 \mathrm{~nm}$, dashed purple line) of $\mathrm{H}_{2} \mathrm{TOFP}$ in $\mathrm{CH}_{2} \mathrm{Cl}_{2}$ at $298 \mathrm{~K}$.)

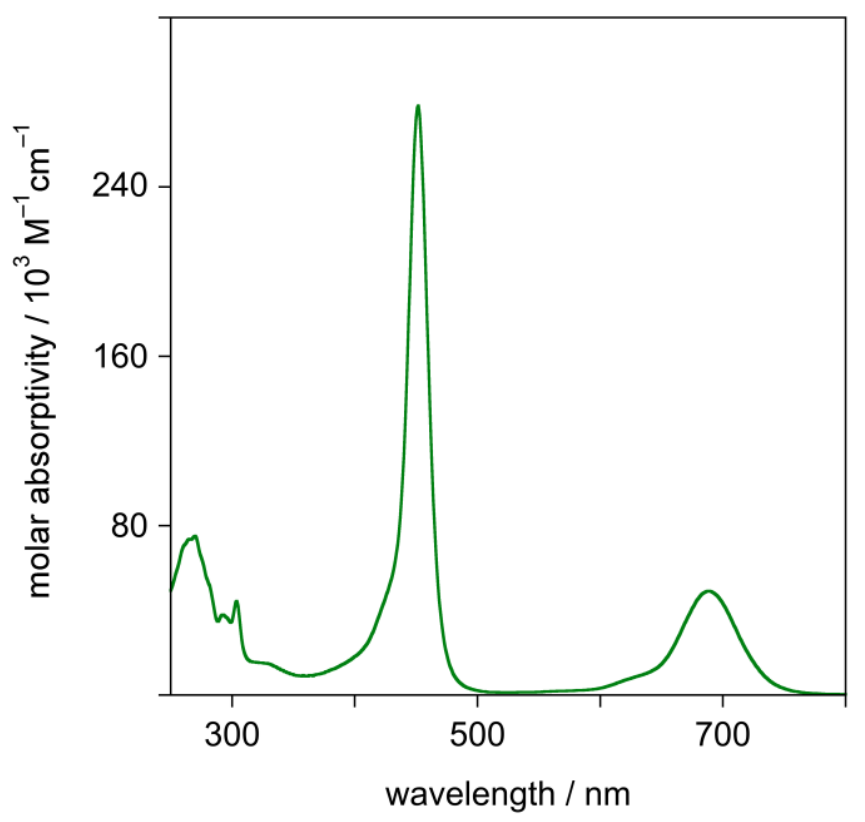

<Figure 3: UV-visible absorption spectrum of $\mathrm{H}_{2}$ TOFP in $\mathrm{CH}_{2} \mathrm{Cl}_{2}$ in the presence of added $\mathrm{H}^{+}$ to give the protonated form, showing a Soret band at $450 \mathrm{~nm}$.> 

in $\mathrm{CH}_{2} \mathrm{Cl}_{2}$ at $298 \mathrm{~K}$

\begin{tabular}{|c|c|c|c|c|}
\hline & $\begin{array}{c}\lambda_{\max }^{\mathrm{abs}} / \mathrm{nm} \\
\text { fluorenyl; Soret; Q }\end{array}$ & $\begin{array}{c}\lambda_{\max }^{\mathrm{em}} / \mathrm{nm} \\
298 \mathrm{~K}\end{array}$ & $298 \mathrm{~K}$ & $298 \mathrm{~K}$ \\
\hline $\mathrm{H}_{2}$ TPP & $---; 417 ;$ & 650,714 & 8.6 & 13 \\
& 513,$548 ; 589 ; 646$ & & & \\
\hline $\mathrm{H}_{2}$ TOFP & $270 ; 422 ;$ & 659,725 & 7.6 & 7.9 \\
& $521,560,592,653$ & & & \\
\hline $\mathrm{H}_{2}$ OOFP & $270 ; 422 ;$ & 657,718 & 7.5 & 11 \\
& $516,550,590,648$ & & & \\
\hline
\end{tabular}

(a) Lifetimes measured by time-correlated single photon counting; estimated error in $\tau$ is $\pm 0.3 \mathrm{~ns}$.

(b) Fluorescence quantum yield measured using tetraphenylporphyrin as the standard; estimated error in $\Phi$ is $\pm 20 \%$.

The emission spectra of the free-base porphyrins in solution display the typical porphyrin-based fluorescence in the red region expected for a tetra-aryl porphyrin (Figure 2, and Table 1). The fluorescence lifetimes of the porphyrins are around $7-8 \mathrm{~ns}$ (e.g., Figure 4), similar to that of $\mathrm{H}_{2}$ TPP. The emission spectra are essentially independent of the excitation wavelength: excitation at short wavelength into the fluorene bands gives the porphyrin emission, with no detectable fluorene emission at higher energy. Likewise, the excitation spectra registered around $660 \mathrm{~nm}$ show bands attributable to excitation of the fluorenes at similar relative intensities to the fluorene bands in the absorption spectrum. Thus, we can conclude that energy transfer from the fluorene pendants to the porphyrin core occurs on a timescale that greatly exceeds that of fluorene fluorescence. Inspection of the emission spectrum of fluorene and the absorption spectrum of $\mathrm{H}_{2}$ TPP (as a model for the "core" of the porphyrin) reveals significant overlap (Figure 5), which will facilitate the energy transfer process from the fluorene pendants to the porphyrin. Although the extinction coefficient of $\mathrm{H}_{2}$ TPP in the $300-350 \mathrm{~nm}$ region is much lower than that of the Soret band, the absorbance is nevertheless strong here $\left(\varepsilon \sim 12000 \mathrm{M}^{-1} \mathrm{~cm}^{-1}\right)$. As anticipated (vide supra), there is essentially no difference in the excited state energies of $\mathrm{H}_{2} \mathrm{OOFP}$ and $\mathrm{H}_{2}$ TOFP, despite the meta versus para linkage of the fluorenyl groups on the pendent aryl rings. 

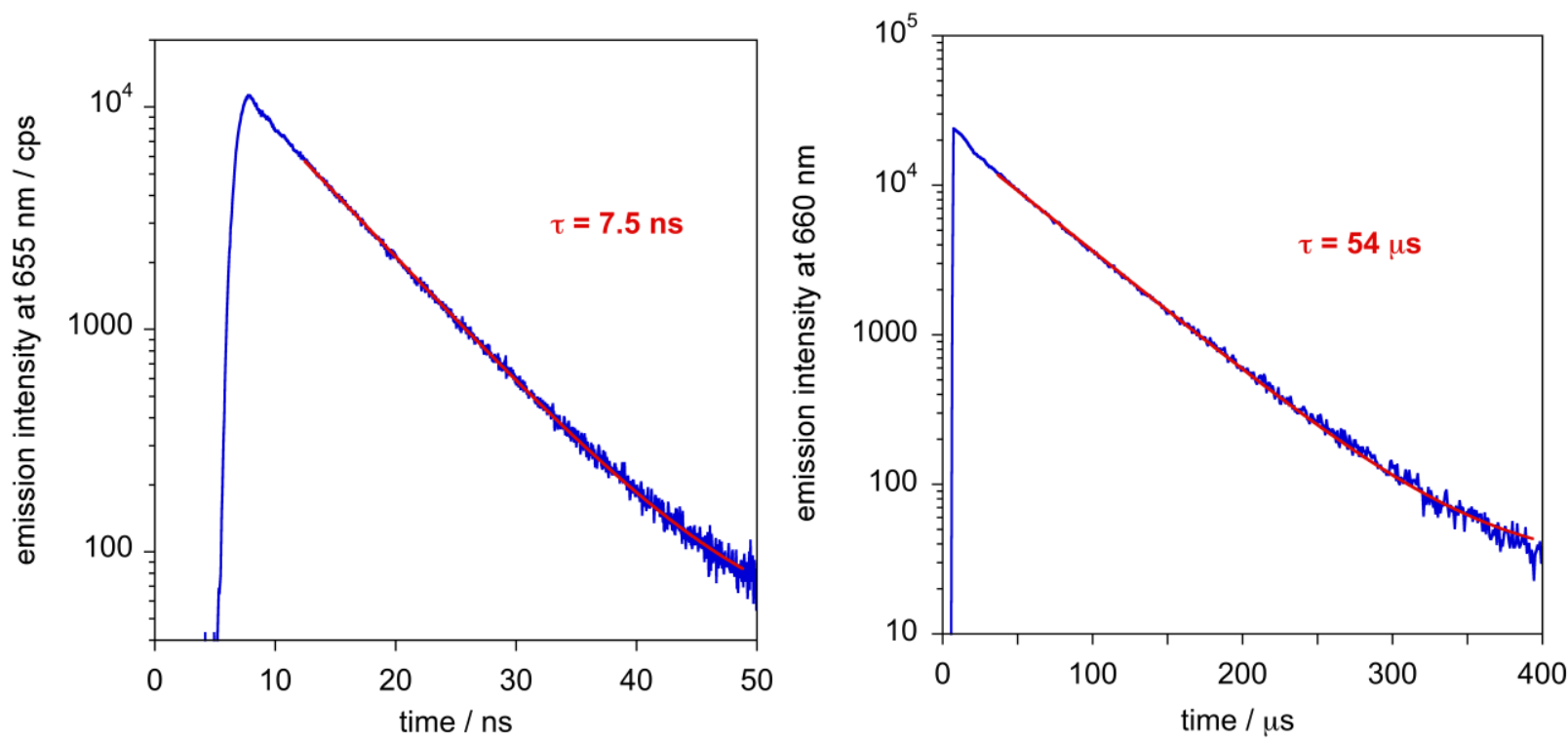

$<$ Figure 4: Temporal fluorescence decay traces for $\mathrm{H}_{2} \mathrm{OOFP}$ (left; $x$ axis in nanoseconds) and PtOOFP (right; $x$ axis in microseconds).>

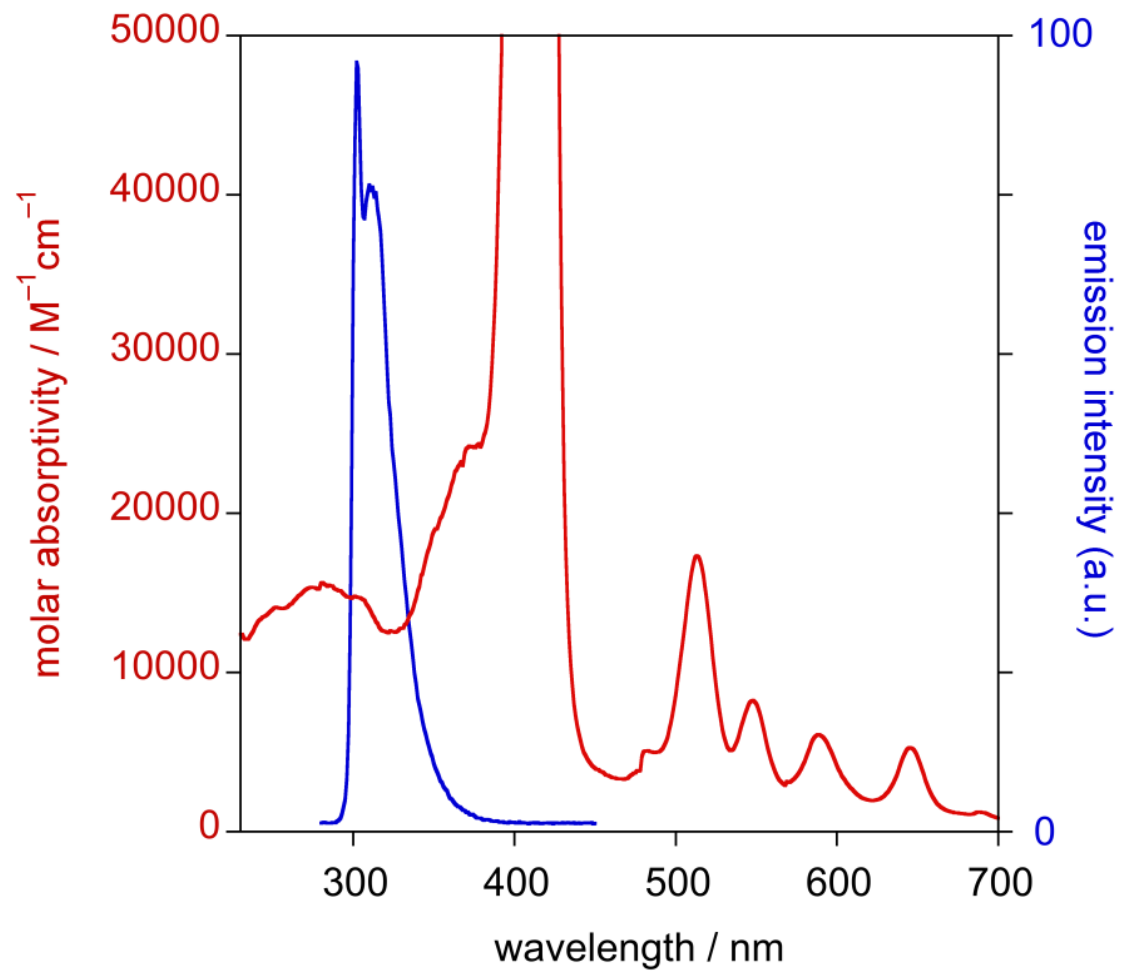

<Figure 5: The emission spectrum of fluorene (blue line) and the absorption spectrum of $\mathrm{H}_{2} \mathrm{TPP}$ (red line) in $\mathrm{CH}_{2} \mathrm{Cl}_{2}$, showing the spectral overlap in the $300-350 \mathrm{~nm}$ region> 
Table 2 Absorption and emission data for the $\mathrm{Pt}$ porphyrins in $\mathrm{CH}_{2} \mathrm{Cl}_{2}$ at $298 \mathrm{~K}$ or $77 \mathrm{~K}$

\begin{tabular}{|c|c|c|c|c|c|c|c|}
\hline & $\begin{array}{c}\lambda_{\max }^{\text {abs }} / \mathrm{nm} \\
\text { fluorenyl; Soret; Q }\end{array}$ & $\begin{array}{c}\lambda_{\max }^{\mathrm{em}} / \mathrm{nm} \\
298 \mathrm{~K}\end{array}$ & $\begin{array}{c}\tau / \mu \mathrm{s}^{(a)} \\
298 \mathrm{~K}\end{array}$ & $\begin{array}{c}\Phi_{\text {lum }} \times 10^{2} \\
298 \mathrm{~K}^{(b)}\end{array}$ & $\begin{array}{c}k_{\mathrm{Q}}^{\mathrm{O} 2(c)} / \\
10^{8} \mathrm{M}^{-1} \mathrm{~s}^{-1}\end{array}$ & $\begin{array}{c}\lambda_{\max }^{\mathrm{em}} / \mathrm{nm} \\
77 \mathrm{~K}\end{array}$ & $\begin{array}{c}\tau / \mu \mathrm{s}^{(a)} \\
77 \mathrm{~K}\end{array}$ \\
\hline PtTPP & $\begin{array}{l}---; 400 ; \\
508,538\end{array}$ & 668,734 & 59 & 4.6 & 6.8 & 655,728 & 132 \\
\hline PtTOFP & $\begin{array}{c}267 ; 406 ; \\
511\end{array}$ & 683,743 & 48 & 11 & 3.3 & 669,741 & 105 \\
\hline PtOOFP & $\begin{array}{c}267 ; 406 ; \\
511,541,599\end{array}$ & 664,729 & 54 & 4.2 & 0.63 & 652,722 & 123 \\
\hline
\end{tabular}

(a) Lifetimes measured by multi-channel scaling; estimated error in $\tau$ is $\pm 4 \mu \mathrm{s}$.

(b) Phosphorescence quantum yield measured using $\mathrm{Ru}(\mathrm{bpy})_{3} \mathrm{Cl}_{2}$ as the standard; estimated error in $\Phi$ is $\pm 20 \%$.

(c) $k_{\mathrm{Q}}$ is the bimolecular rate constant for quenching by molecular oxygen, estimated from the lifetimes in degassed and aerated solution, and assuming that $\left[\mathrm{O}_{2}\right]=2.2 \mathrm{mmol} \mathrm{dm}^{-3}$ in $\mathrm{CH}_{2} \mathrm{Cl}_{2}$ at $298 \mathrm{~K}$ and $1 \mathrm{~atm}$ pressure air.

(ii) Platinum porphyrins. Metallation of the porphyrins is accompanied by a blue-shift in the Soret and Q bands, as typically observed for metalloporphyrins (Figure 6 and Table 2). The number of $\mathrm{Q}$ bands is reduced to two, in line with the expected $D_{4 \mathrm{~h}}$ symmetry. The platinum(II) complexes of the porphyrins display emission bands deeper into the red than the free-base analogues, with long lifetimes of around $50 \mu \mathrm{s}$, indicative of emission emanating from the triplet excited state (Figure 6 and Table 2). In this case, there is slightly more difference in energy between the Pt(II) complexes of TOFP and OOFP than between the corresponding free-base porphyrins, suggesting that the triplet state is influenced slightly more than the singlet by the aryl substitution pattern. Again, no fluorene emission is detected upon excitation at high energy into the fluorene bands, confirming the fast energy transfer to the metalloporphyrin unit. Efficient energy transfer is also confirmed by good superposition of absorption spectrum (black line) and the excitation spectrum (dashed purple line) in Figure 6. The phosphorescence quantum yields are of the order of $0.04-0.10$ (Table 2), and the emission is quenched efficiently by dissolved $\mathrm{O}_{2}$. The bimolecular rate constants for diffusional-controlled oxygen quenching are in the order PtTPP > PtTOFP > PtOOFP, the values decreasing as the molecular weights increase (and hence as diffusion coefficients decrease). At $77 \mathrm{~K}$, the emission spectra become somewhat sharper, as the environment becomes more rigid, although with scarcely any shift in the maxima. Such behaviour is quite typical for $\pi-\pi^{*}$ states in highly symmetric luminophores. The lifetimes at 77 
$\mathrm{K}$ are increased by a factor of around 2 compared to room temperature values (Table 2), reflecting the partial inhibition of non-radiative decay processes under these conditions.

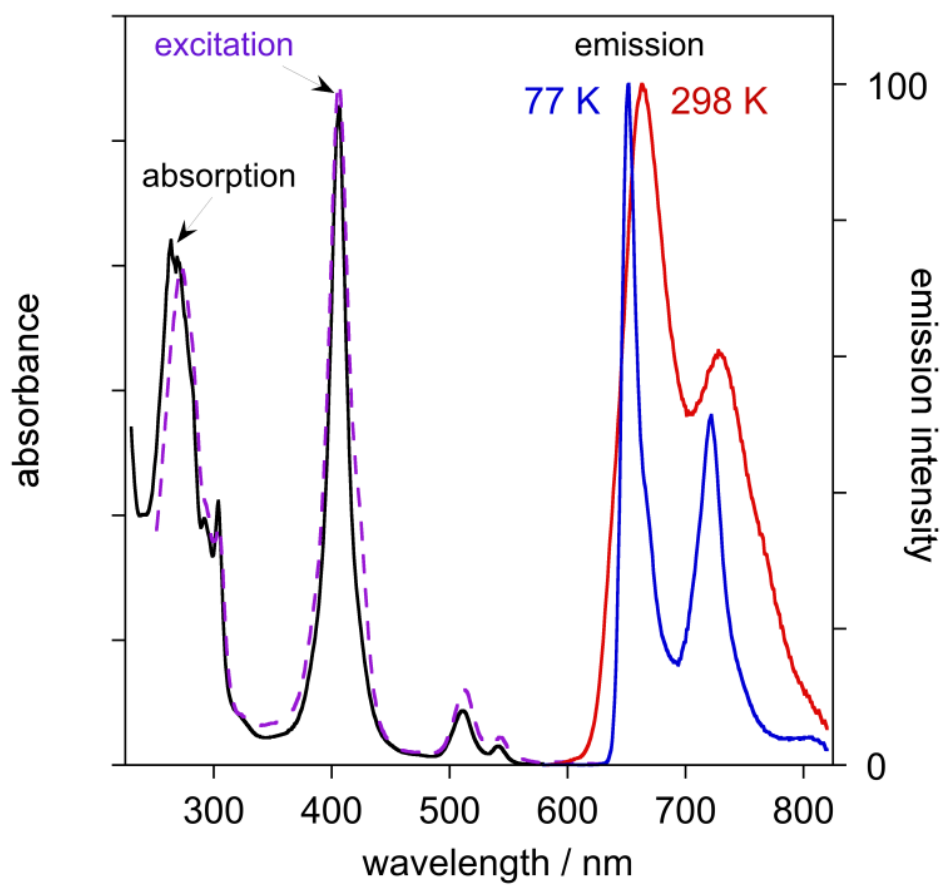

< Figure 6: UV-visible absorption spectrum (black line), and excitation spectrum $\left(\lambda_{\text {em }}=660\right.$ $\mathrm{nm}$, dashed purple line) and emission spectrum (red line) of PtOOFP in $\mathrm{CH}_{2} \mathrm{Cl}_{2}$ at $298 \mathrm{~K}$.

The emission spectrum at $77 \mathrm{~K}$ is also shown (blue line).>

\section{Electronic and optoelectronic behaviour of OLEDs}

The complexes were incorporated into OLEDs followed a procedure previously described. ${ }^{22}$ Initially, a layer of poly(3,4-ethylenedioxythiophene)/poly(styrenesulfonate) (PEDOT/PSS) was spin-coated onto the indium tin oxide (ITO) layer on a glass substrate, followed by a blended emissive layer. This consisted of the host, polyvinylcarbazole (PVK), together with the platinum complex (3\% by weight) and 1,3-bis(4-tert-butylphenyl-1,3,4-oxadiazolyl) phenylene (30\% by weight), denoted OXD7 (also called OXA, structure in Figure 7), as an electron transport layer. This was also deposited by spin-coating, using chlorobenzene or chloroform as a solvent. Finally, a thin caesium fluoride layer and then the aluminium cathode were thermally evaporated under vacuum. In such a blended OLED structure, the emitting layer is formed from a single spin-coated layer. Devices should therefore be easy to fabricate. ${ }^{23}$ 
Electron transporting layer used:

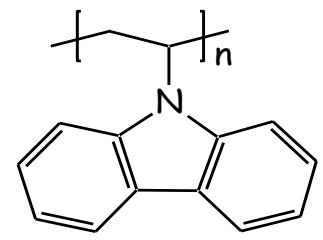

PVK (Host Matrix)

PolyVinylCarbazole
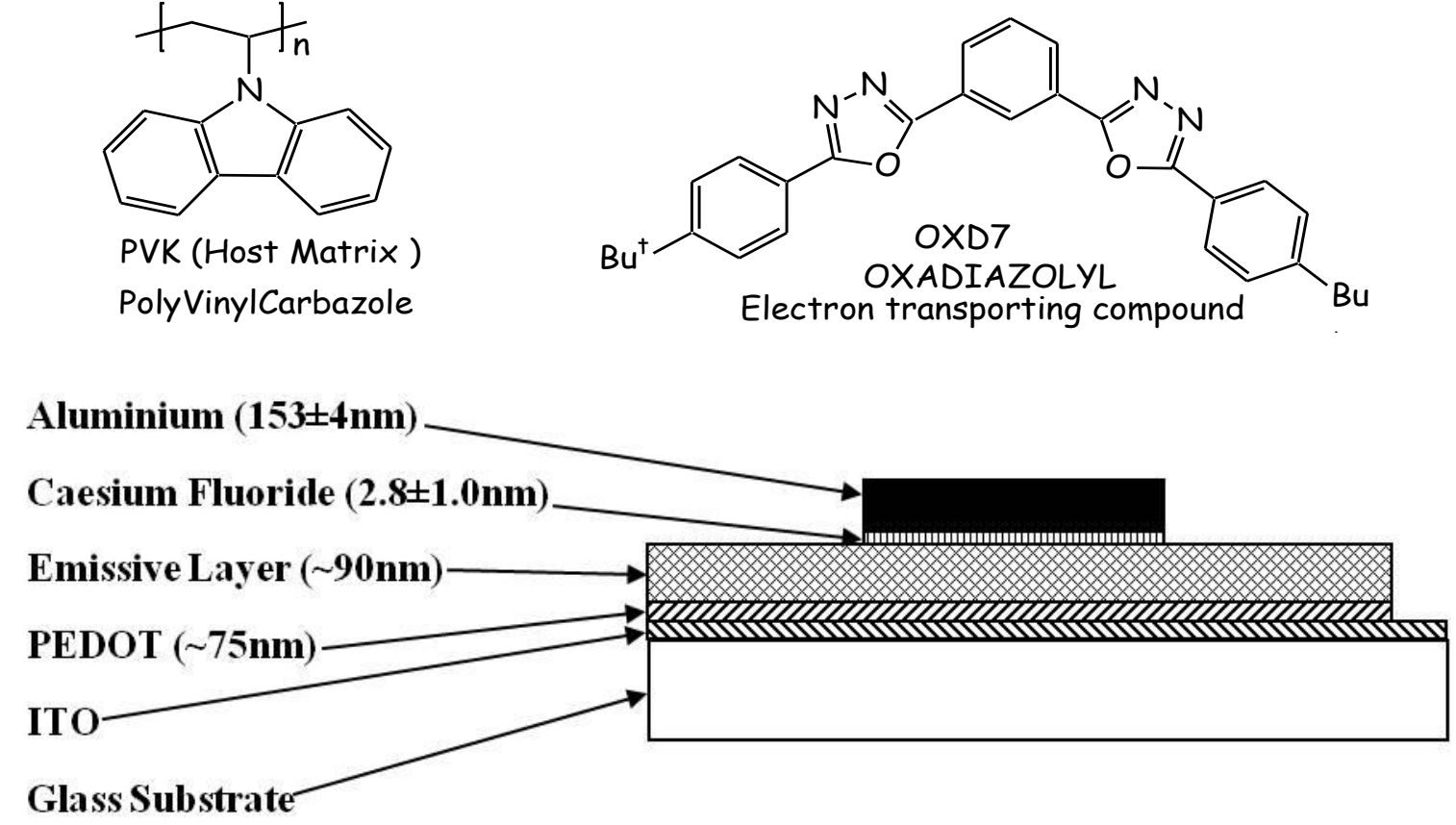

$<$ Figure 7: Schematic diagram of the OLED configuration used in this work

(PEDOT noted for PEDOT/PSS layer).>

The normalised electroluminescence (EL) spectra are shown in Figure 8 for the set of devices having the configurations shown in the caption to the Figure. The spectra were measured at a current of $1 \mathrm{~mA}$ (current density $5.1 \mathrm{~mA} \mathrm{~cm}^{-2}$ ). Emission from the PVK-only control device (device i) gave a maximum at $412 \mathrm{~nm}$. The addition of OXD7 to give device (ii) resulted in a broader EL spectrum with a maximum at $468 \mathrm{~nm}$. This can be related to the formation of an exciplex between the two components (i.e., the coupling of an excited singlet state of one molecule with the ground state of another). The elaboration of device (iii) was done by addition of PtOOFP to PVK-OXD7; for this device, a broad emission from 400 to $600 \mathrm{~nm}$ was observed, with peaks at $470 \mathrm{~nm}$ (from the PVK-OXD7 exciplex) and $540 \mathrm{~nm}$ and a shoulder at $516 \mathrm{~nm}$. For the device (iv), when chloroform was used as the spin-coating solvent, peaks at 544 and 576 $\mathrm{nm}$ and shoulders at 460 and $512 \mathrm{~nm}$ were observed. In addition, both devices (iii) and (iv) exhibited intense red EL bands at around 668 and $730 \mathrm{~nm}$. The device containing our previously reported PtTFP (v) exhibited a broad emission at $468 \mathrm{~nm}$ (from the PVK-OXD7 exciplex) with maximum red EL at $680 \mathrm{~nm}$ and a second peak at $744 \mathrm{~nm}$. The emission from the PVK-OXD exciplex suggests that there is only partial energy transfer between the host matrix and the guest dye in this device, inferior to those with the new porphyrins. Finally, the control device (vi) containing PtTPP in place of the fluorenyl porphyrins exhibited only red emission, with a 
maximum EL at $668 \mathrm{~nm}$ and a second peak at $732 \mathrm{~nm}$. The red emission peaks from these devices agree well with those observed in our previous work. ${ }^{20}$

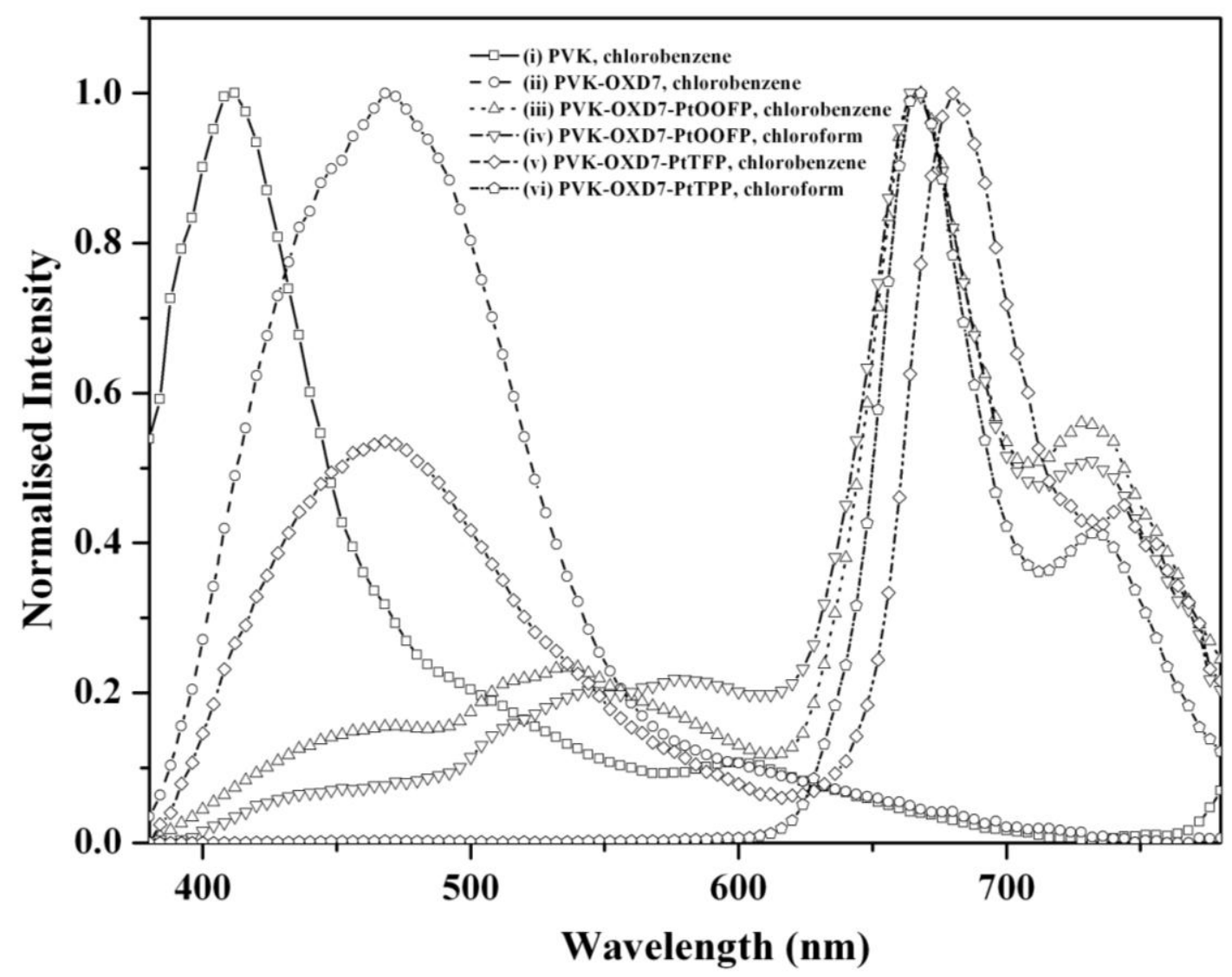

< Figure 8: Normalised EL spectra of OLEDs with the following configurations:

(i) ITO/PEDOT-PSS/PVK/CsF/Al, chlorobenzene solvent (full line);

(ii) ITO/PEDOT-PSS /PVK-OXD7/CsF/Al, chlorobenzene solvent (dashed line);

(iii) ITO/PEDOT-PSS /PVK-OXD7-PtOOFP/CsF/Al, chlorobenzene solvent (dotted line);

(iv) ITO/PEDOT-PSS /PVK-OXD7-PtOOFP/CsF/Al, chloroform solvent (dash-dotted line);

(v) ITO/PEDOT-PSS /PVK-OXD7-PtTFP/CsF/Al, chlorobenzene solvent (dash-dot-dotted line);

(vi) ITO/PEDOT-PSS/PVK-OXD7-PtTPP/CsF/Al, chloroform solvent (short dash-dotted line).>

The "Commission Internationale de I'Eclairage" (CIE) coordinates corresponding to the spectra shown in Figure 8 are plotted in Figure 9. The D65 white point is also included on the diagram as a reference. The EL from the PVK (i) and PVK-OXD7 (ii) devices was blue in colour, as was that from the device containing PtTFP (v) (due to the broad emission from the PVK-OXD7 exciplex). The broad blue/green emissions combined with the red peaks in the spectra of the devices containing PtOOFP (iii and iv) rendered EL from these devices white in appearance (particularly the device prepared from chlorobenzene solvent). The emission from the device containing PtTPP (vi) was confined to the long wavelength part of the spectrum, giving a red colour. 
The turn-on voltages (bias required to produce a current of $1 \mathrm{nA}$ from the photodiode detector), current, and power efficiencies are presented in Table 3. Surprisingly, the turn-on voltages for the two devices containing PtOOFP, (iii) and (iv), differ substantially, despite them being identical other than in the solvent used for preparation. The device where chlorobenzene was used displays a turn-on voltage of $6.9 \mathrm{~V}$, compared to $21.9 \mathrm{~V}$ for the chloroform analogue, similar to the device containing PtTPP (vi). For the device containing PtTFP (v), the value is quite low $(8.2 \mathrm{~V})$. The differing performance of the OLEDs according to the identity of the solvent used for the spin-coating process is surprising. Given that chlorobenzene is an aromatic solvent whereas chloroform is not, it seems likely that the reason for the differing behaviour may arise from the porphyrin molecules adopting a different packing arrangement relative to one another according to the solvent from which evaporation occurs. The precise arrangement of molecules may strongly influence intermolecular interactions and hence both the chargetransporting and excited state quenching processes. Such effects have been observed and examined in detail for simpler aromatic molecules such as anthracene. ${ }^{24}$

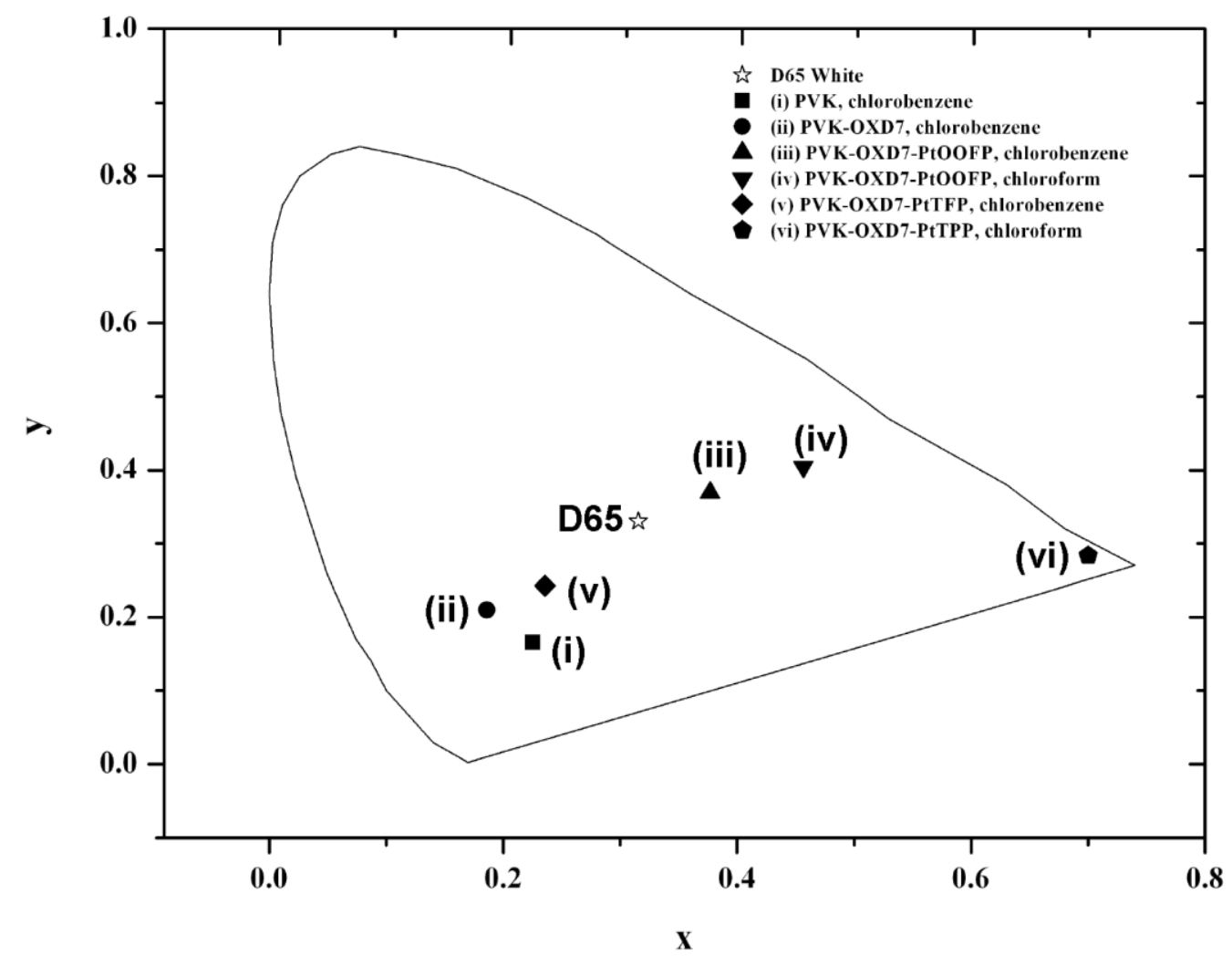

$<$ Figure 9: CIE coordinates for the OLEDs prepared here, the EL spectra of which are shown in Fig 5. >

Table 3 Electroluminescence data for OLEDs (i) to (vi). Turn-on voltages given at $1 \mathrm{nA}$ of photocurrent, as measured by the photodiode detector. All other data were measured at a current of $1 \mathrm{~mA}$ (current density $5.1 \mathrm{~mA} \mathrm{~cm}$ ). 


\begin{tabular}{|c|c|c|c|c|c|c|}
\hline Device & (i) & (ii) & (iii) & (iv) & (v) & (vi) \\
\hline Turn on voltage (V) & 5.9 & 5.5 & 6.9 & 21.9 & 8.2 & 21.5 \\
\hline Current efficiency $\left(\mathrm{cd} \mathrm{A}^{-1}\right)$ & 0.21 & 0.29 & 0.27 & 0.18 & 0.11 & 0.14 \\
\hline Power efficiency $\left(\mathrm{lm} \mathrm{W}^{-1}\right)$ & 0.06 & 0.06 & 0.06 & 0.01 & 0.03 & 0.01 \\
\hline $\mathrm{CIE}_{\mathrm{x}}$ & 0.219 & 0.179 & 0.372 & 0.453 & 0.230 & 0.699 \\
\hline $\mathrm{CIE}_{\mathrm{y}}$ & 0.166 & 0.210 & 0.369 & 0.405 & 0.242 & 0.283 \\
\hline
\end{tabular}

\section{Conclusions}

In summary, we have synthesised and characterised new platinum(II) porphyrin complexes PtTOFP and PtOOFP bearing four and eight fluorenyl pendant arms at the meso-positions, respectively. These platinum(II) complexes PtTOFP and PtOOFP were shown to emit essentially red light after selective UV irradiation and visible irradiation. We have also investigated the electronic and optoelectronic behaviour of OLEDs based on these red phosphorescent dyes, as well as earlier reported PtTFP and reference PtTPP, doped into a layer of PVK host. The EL from the device containing PtTFP was blue in colour, due to the residual emission from the PVK-OXD7 exciplex. Energy transfer from the host appears to be more efficient using the new octafluorenylporphyrin, and the two devices containing PtOOFP gave the EL a white appearance. These OLEDs have been obtained using a relatively simple device configuration, which should be comparatively easy to manufacture. However, it is clear that the choice of solvent used in the preparation of the device can influence the device characteristics, including a subtle effect on the colour and a much more significant effect on the turn-on voltage. The choice of meso-aryl substituents can allow the properties of porphyrins to be optimised for OLEDs. Accordingly, these new platinum fluorenyl-appended complexes constitute interesting building blocks to access new emissive materials.

\section{Experimental section}

\section{General}

All reactions were performed under argon and were magnetically stirred. Solvents were distilled from appropriate drying agent prior to use, $\mathrm{CH}_{2} \mathrm{Cl}_{2}$ from $\mathrm{CaH}_{2}, \mathrm{CHCl}_{3}$ from $\mathrm{P}_{2} \mathrm{O}_{5}$ and all other solvents were HPLC grade. Pyrrole, trifluoroacetic acid and aldehyde were purchased from Aldrich and were used as received. Commercially available reagents were used without further purification unless otherwise stated. All reactions were monitored by thin layer chromatography (TLC) with Merck pre-coated aluminium foil sheets (Silica gel 60 with fluorescent indicator 
$\mathrm{UV}_{254}$ ). Compounds were visualised with ultra-violet (UV) light at $254 \mathrm{~nm}$ and $365 \mathrm{~nm}$. Column chromatography was carried out using silica gel from Merck (0.063-0.200 mm). Nuclear Magnetic Resonance (NMR) spectroscopy in $\mathrm{CDCl}_{3}$ was recorded using Bruker 200 DPX spectrometers, the chemical shifts are referenced to an internal tetramethylsilane (TMS) standard.

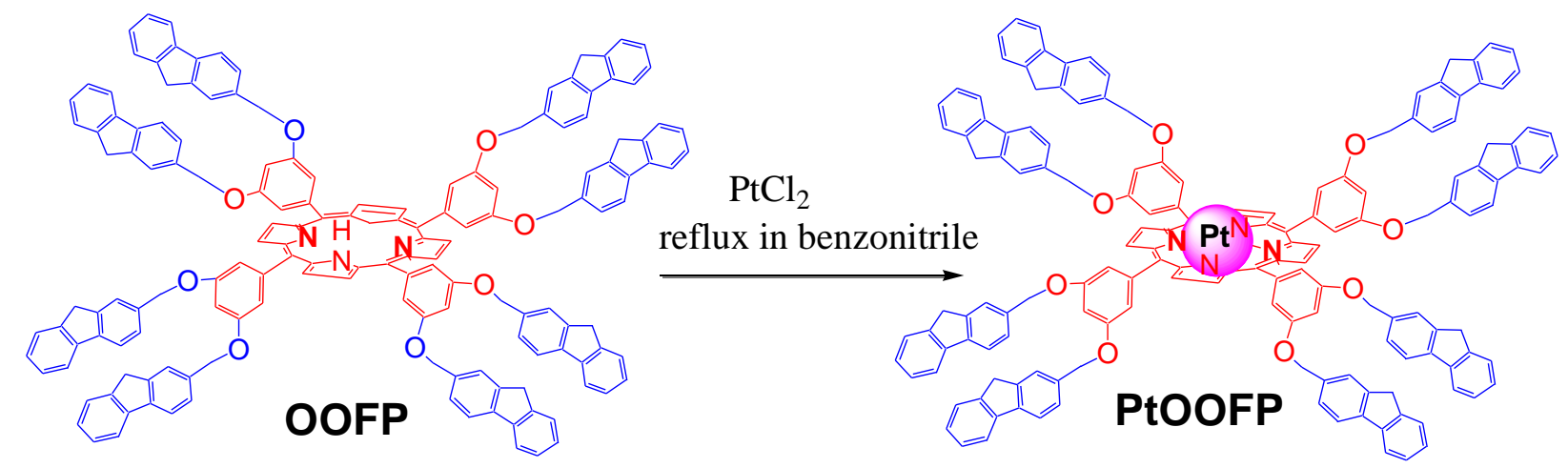

Scheme 1 Synthesis of metalloporphyrins.

\section{Synthesis of metalloporphyrins (Scheme 1)}

Meso-(5,10,15,20-tetra(2 fluorenyl)porphyrinato platinum(II) (PtTFP) was prepared by an adaptation of the procedure used for the Pt(II) complex of TPP, ${ }^{21}$ as described recently by our group. ${ }^{20}$ A suspension of $\mathrm{PtCl}_{2}(0.17 \mathrm{~g}, 0.64 \mathrm{mmol})$ in benzonitrile $(20 \mathrm{~mL})$ was pre-heated at $100^{\circ} \mathrm{C}$ for $1 \mathrm{~h}$ under an argon atmosphere. Free-base porphyrin TFP $(0.15 \mathrm{~g}, 0.155 \mathrm{mmol})$ was added and the mixture refluxed for $7 \mathrm{~h}$, the progress of the reaction being monitored by TLC. The solvent was then removed by vacuum distillation, and the residue purified by chromatography (silica gel, dichloromethane/pentane $=10 / 1$ ) to give the desired product as a reddish-purple solid (120 mg, 67\%). This soluble porphyrin complex PtTFP was identified by ${ }^{1} \mathrm{H}$

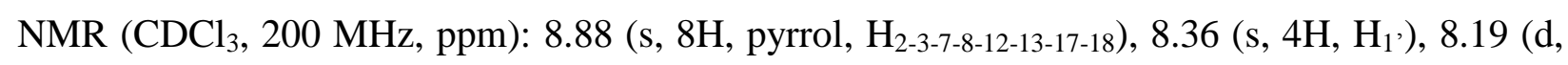
$\left.{ }^{3} \mathrm{~J}_{\mathrm{HH}}=8.2,4 \mathrm{H}, \mathrm{H}_{3^{\prime}}\right), 8.08\left(\mathrm{~d},{ }^{3} \mathrm{~J}_{\mathrm{HH}}=8.2,4 \mathrm{H}, \mathrm{H}_{4}{ }^{\prime}\right), 7.84\left(\mathrm{~d},{ }^{3} \mathrm{~J}_{\mathrm{HH}}=6.6,4 \mathrm{H}, \mathrm{H}_{5}{ }^{\prime}\right), 7.74\left(\mathrm{~m}, 4 \mathrm{H}, \mathrm{H}_{8^{\prime}}\right)$, 7.53-7.46 (m, 8H, $\mathrm{H}_{6}$, and $\left.\mathrm{H}_{7}{ }^{\prime}\right), 4.23$ (s, 8H, $\left.\mathrm{H}_{9}{ }^{\prime}-9^{\prime}\right)$.

The platinum(II) complexes of TOFP and OOFP (see scheme 1) were prepared as previously published to obtain porphyrin platinum complexes. ${ }^{21,25}$

\section{Meso-(5,10,15,20-tetra(4-(2 methyloxyfluorenyl)phenyl)porphyrinato platinum(II) (PtTOFP)}

was prepared by platinum insertion in TOFP following an adapted procedure of the platinum insertion in TPP. ${ }^{21} \mathrm{PtCl}_{2}(0.04 \mathrm{~g}, 0.15 \mathrm{mmol})$ was dissolved in $12 \mathrm{~mL}$ of purified benzonitrile and preheated at $100{ }^{\circ} \mathrm{C}$ for $1 \mathrm{~h}$ under argon atmosphere. Free-base porphyrin TOFP $(0.05 \mathrm{~g}$, 
$0.04 \mathrm{mmol}$ ) was added to the previous solution and the mixture was refluxed for another $4 \mathrm{~h}$. Reaction progress is monitored by TLC, spotting directly from the organic layer. This mixture was cooled to room temperature and the precipitate collected by filtration. The precipitate was washed thoroughly with $\mathrm{MeOH}$ and $\mathrm{CH}_{2} \mathrm{Cl}_{2}$ to give the desired product (yield 90\%) as a reddishpurple solid. This relatively insoluble porphyrin complex PtTOFP was characterised by NMR, mass spectrometry and UV-Vis. ${ }^{1} \mathrm{H}$ NMR $\left(\mathrm{CDCl}_{3}, 200 \mathrm{MHz}, \mathrm{ppm}\right): 8.80$ (large s, 8H, pyrrol, $\mathrm{H}_{2-}$ 3-7-8-12-13-17-18), 8.40-7.40 (m, 44H, $\mathrm{H}_{\text {fluorenyl-phenyl }}$ ), 5.30 (s, 8H, $\mathrm{H}_{\mathrm{H}-\mathrm{H}^{\prime}}$ ), 3.90 (large s, 8H, $\mathrm{H}_{9}$-9'). MS (Maldi -TOF): calcd for $\mathrm{C}_{100} \mathrm{H}_{68} \mathrm{~N}_{4} \mathrm{O}_{4} \mathrm{Pt}: 1583.4900[\mathrm{MH}]^{+}$, found: $1583.4170[\mathrm{MH}]^{+}$. UVvisible (in $\mathrm{CH}_{2} \mathrm{Cl}_{2}$ ): $267 \mathrm{~nm}$ (fluorene), $406 \mathrm{~nm}$ (Soret band), $511 \mathrm{~nm}$ (Q band).

\section{Meso-(5,10,15,20-tetra(4-(3,5 dimethyloxyfluorenyl)phenyl)porphyrinato platinum(II) (PtOOFP)} was prepared by platinum insertion in OOFP, following the same procedure. ${ }^{21} \mathrm{PtCl}_{2}(0.104 \mathrm{~g}$, $0.39 \mathrm{mmol}$ ) was dissolved in $20 \mathrm{~mL}$ of purified benzonitrile and preheated at $100{ }^{\circ} \mathrm{C}$ for $1 \mathrm{~h}$ under argon atmosphere. Free-base porphyrin OOFP $(0.20 \mathrm{~g}, 0.09 \mathrm{mmol})$ was added to the previous solution and the mixture was refluxed for another $2 \mathrm{~h}$. Reaction progress is monitored by TLC, spotting directly from the organic layer. This mixture was condensed by vacuum distillation and cooled to room temperature. The crude product was chromatographed on silica gel and eluted with (10:1 dichloromethane/methanol) to give the desired product (yield 95\%) as a reddish-purple solid. This soluble porphyrin complex PtOOFP was characterised by NMR, mass spectrometry, micro-analysis and UV-Vis. The hydrogen atom-labelling scheme for the porphyrin ligand is shown in Figure 10. ${ }^{1} \mathrm{H}$ NMR $\left(\mathrm{CDCl}_{3}, 200 \mathrm{MHz}, \mathrm{ppm}\right): 8.77$ (s, 8H, pyrrol,

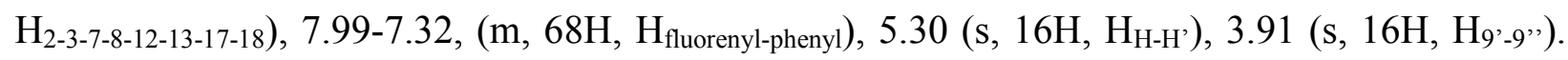
UV-visible (in $\mathrm{CH}_{2} \mathrm{Cl}_{2}$ ): $267 \mathrm{~nm}$ (fluorene), $406 \mathrm{~nm}$ (Soret band), 511, 541, $599 \mathrm{~nm}$ (Q bands). MS (Maldi -TOF): calcd for $\mathrm{C}_{156} \mathrm{H}_{106} \mathrm{~N}_{4} \mathrm{O}_{8} \mathrm{Pt}$ : $2358.7700[\mathrm{MH}]^{+}$, found: $2358.2260[\mathrm{MH}]^{+}$. Analysis: calcd for $\mathrm{C}_{156} \mathrm{H}_{106} \mathrm{~N}_{4} \mathrm{O}_{8} \mathrm{Pt} .2 \mathrm{CH}_{2} \mathrm{Cl}_{2}$ : C, 75.02; H, 4.38; N, 2.21 found C, 75.47; H, 4.94; $\mathrm{N}, 2.22$. 


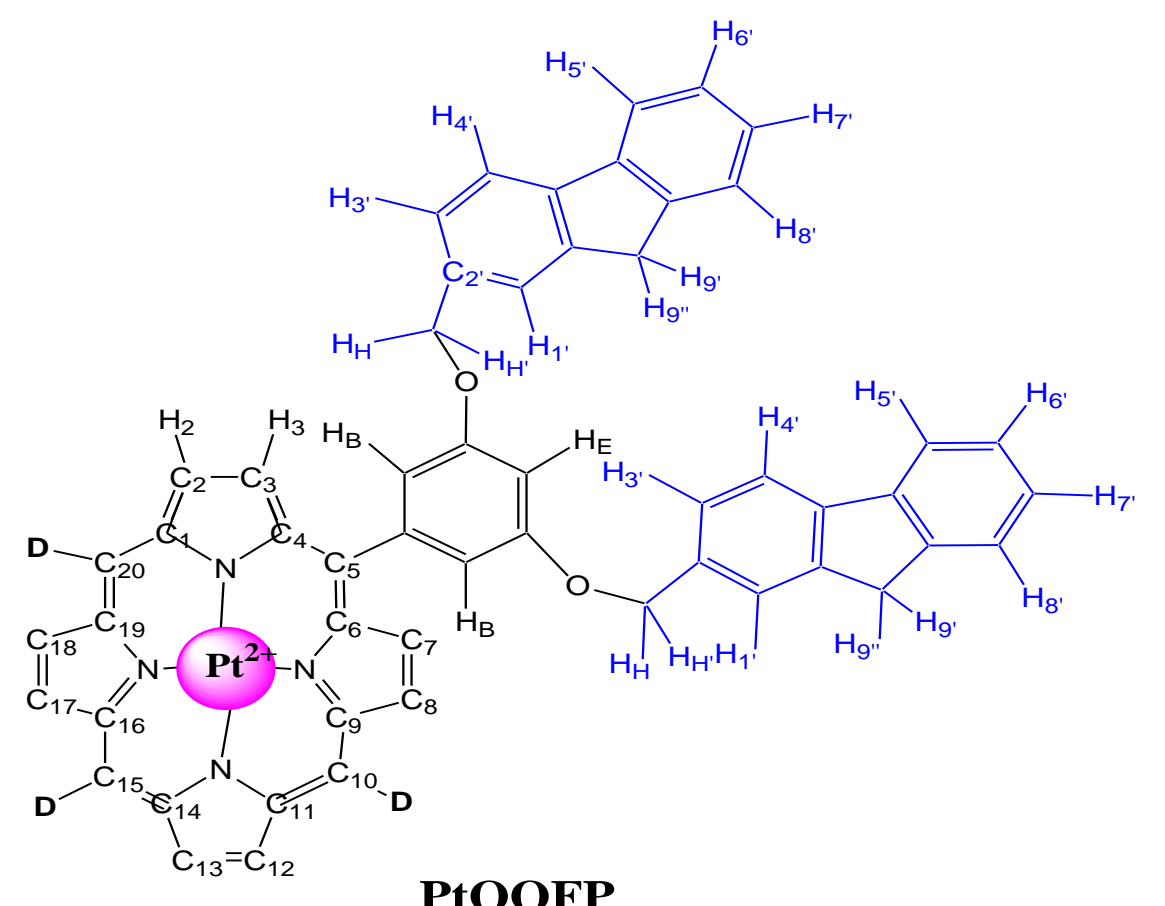

< Figure 10: Hydrogen and carbon atom-labelling for the porphyrin complex PtOOFP.>

\section{Absorption and emission spectroscopy in solution}

UV-vis spectra in dichloromethane solution were recorded on a Biotek Instruments XS spectrophotometer, using $1 \mathrm{~cm}$ pathlength quartz cuvettes. The steady-state fluorescence spectra were measured at $298 \mathrm{~K}$ using a Jobin Yvon Fluoromax-2 spectrofluorimeter, equipped with a red-sensitive Hamamatsu R928 photomultiplier tube. The emission spectra were corrected for the wavelength dependence of the PMT/emission monochromator by application of a correction curve generated from a standard lamp. Quantum yields were obtained by standard serial dilution methods at high dilution, using degassed solutions to limit photo-oxidative degradation. For the free-base porphyrins, a solution of tetraphenylporphyrin in benzene was used as the standard $\left(\Phi_{\mathrm{F}}\right.$ $=0.11^{26}$ ) and correction made for the difference in refractive indices of dichloromethane and benzene $(n=1.421$ and 1.501 , respectively). For the platinum complexes, an aqueous solution of ruthenium(II) tris-bipyridine dichloride was used as the standard $\left(\Phi_{\mathrm{F}}=0.042^{27} ; \mathrm{n}=1.333\right)$. The estimated error on quantum yields is $\pm 20 \%$, and values are therefore quoted to two significant figures. The fluorescence lifetimes of the free-base porphyrins were measured by timecorrelated single-photon counting, exciting at $374.0 \mathrm{~nm}$ with an EPL-375 pulsed diode laser at a repetition rate of $1 \mathrm{MHz}$ (pulse length ca. $60 \mathrm{ps}$ ), and detecting the emitted light at $90^{\circ}$ after passage through a monochromator using a Peltier cooled R928. The lifetimes were extracted from the measured decays by reconvolution of the instrument response, obtained using a suspension of Ludox ${ }^{\circledR}$ in water as a non-fluorescent scatterer. The phosphorescence lifetimes of 
the platinum porphyrins were obtained by multi-channel scaling of the emitted light using the same detector, following excitation with a microsecond pulsed xenon lamp.

\section{Electronic and optoelectronic studies of OLEDs}

A schematic diagram of the device structure used in this investigation is shown in Figure 4. Devices were fabricated on indium tin oxide (ITO) coated glass supplied by VisionTek Systems, with a sheet resistances of $7 \Omega / \mathrm{sq}$. The substrates were cut to size $(\sim 16 \times 21 \mathrm{~mm})$, before being carefully cleaned using the following procedure: rinsed in a jet of isopropyl alcohol (IPA laboratory reagent grade, Fisher Scientific) from a wash bottle; sonicated in IPA for 15 min; rinsed in a jet of IPA from a wash bottle; dried in a stream of nitrogen gas; rinsed in a jet of acetone (analytical reagent grade, Fisher Scientific) from a wash bottle; sonicated in acetone for 15 min; rinsed in a jet of acetone from a wash bottle; dried in a stream of nitrogen gas; rinsed in a stream of ultrapure water (obtained by reverse osmosis, carbon filtration, two stage deionization, and UV sterilization); sonicated in a solution of Decon 90 detergent (5\% in ultrapure water) for $15 \mathrm{~min}$; rinsed in a stream of ultrapure water; sonicated in ultrapure water for $15 \mathrm{~min}$; rinsed in a stream of ultrapure water; and finally dried in a stream of nitrogen gas.

Device fabrication was performed in an inert gas glove box (Glove Box Technology Ltd.). Cleaned substrates were first spin-coated with an $\sim 75$-nm-thick layer of poly(3,4ethylenedioxythiophene) / poly(styrenesulfonate) (PEDOT/PSS) from aqueous dispersion (CLEVIOS P VP AI 4083, Heraeus, Germany). Before coating, the PEDOT/PSS solution was passed through a $0.2 \mu \mathrm{m}$ syringe filter to remove any residual particulates. A volume of $200 \mu \mathrm{L}$ of the filtered dispersion was applied to the surface of the substrate, which was then spun at 2500 $\mathrm{rpm}$ for $45 \mathrm{~s}$. The coated substrates were annealed at $180^{\circ} \mathrm{C}$ for $2 \mathrm{~min}$ to remove any residual water.

The chemical structures of the various materials that were used to form the blended electroluminescent layer in these devices are shown in Figure 1: PVK (average $M W 1100000$, Aldrich); OXD7; PtOOFP, PtTFP and PtTPP. All compounds were used as supplied, without further purification. Separate chlorobenzene (AnalaR, BDH) or, where a material was insoluble in chlorobenzene, chloroform (Trace analysis grade, Fisher Scientific) solutions of each material were prepared before mixing in the proportions necessary to give a blended solution of the required concentration and composition. The concentrations expressed as percentages throughout this paper are all by weight percent. $150 \mu \mathrm{L}$ of this solution was applied to the surface of the substrate, which was then spun for $60 \mathrm{~s}$ at the speed necessary to give a layer having the desired thickness. The coated substrates were annealed at $80^{\circ} \mathrm{C}$ for $30 \mathrm{~min}$ to remove any residual solvent. 
The final fabrication step was the deposition of a $2 \times 3$ array of $5 \mathrm{~mm}$ diameter caesium fluoride (CsF, 99.99\%, Aldrich) / aluminium (Al, AnalaR, BDH) cathodes by thermal evaporation through a shadow mask. The deposition was carried out under a base vacuum of $\sim 4 \times 10^{-6}$ mbar ( $\sim 3 \times 10^{-6}$ torr). $2.8 \pm 1.0 \mathrm{~nm}$ of CsF was deposited at a rate of $0.20 \pm 0.05 \mathrm{~nm} \mathrm{~s}^{-1}$, followed by $153 \pm 4 \mathrm{~nm}$ of $\mathrm{Al}$ at a rate of $1.0 \pm 0.1 \mathrm{~nm} \mathrm{~s}^{-1}$.

Electrical measurements were carried out under vacuum $\left(10^{-1} \mathrm{mbar}\right)$ in a screened metal chamber. Bias voltages were supplied and sample currents measured using a Keithley model 2400 sourcemeter. The devices were mounted over a large area photodiode and the photocurrent generated as a result of the light output was recorded using a Keithley model 485 digital picoammeter. The instruments were connected via general purpose interface bus (GPIB) to a personal computer, which was used to automate the measurement process. Photometric and colorimetric measurements were performed using a Photo Research Inc. PR-655 SpectraScan spectroradiometer fitted with a FP-655 flexible probe (3.18 mm tip diameter).

\section{Acknowledgments}

This research was supported by grants from the "Région Bretagne" (ARED - A. MERHI), the "Ministère National de la Recherche et de la Technologie » (MNERT - S. DROUET), and EPSRC (DTA studentship to L. F. GILDEA). The authors are grateful to S. Sinbandhit (CRMPO) for technical assistance and helpful discussions.

\section{References}

(1) Sheat, J. R.; Antoniadis, H.; Hueschen, M.; Leonard, W.; Miller, J.; Moon, R.; Roitman, D.; Stocking, A. Science 1996, 273, 884 .

(2) Burroughes, J. H.; Bradley, D. C.; Brown, A. B.; Marks, R. N.; Mackey, K.; Friend, R. H.; Bum, P. L.; Holmes, A. B. Nature 1990, 347, 539.

(3) Kraft, A.; Grimsdale, A. C.; Holmes, A. B. Angew. Chem., Int. Ed. 1998, 37, 402.

(4) Rault-Berthelot, J. Electrochemistry 2003, 10, 265.

(5) Virgili, T.; Lidzey, D. G.; Bradley, D. C. Synth. Metals 2000, 111-112, 203.

(6) Li, B.; Xu, X.; Sun, M.; Fu, Y.; Yu, G.; Liu, Y.; Bo, Z. Macromolecules 2006, 39, 456.

(7) Li, B.; Li, J.; Fu, Y.; Bo, Z. J. Am. Chem. Soc. 2004, 126, 3430.

(8) Paul-Roth, C.; Rault-Berthelot, J.; Simonneaux, G. Tetrahedron 2004, 60, 12169.

(9) Simonneaux, G.; Galardon, E.; Paul-Roth, C.; Gulea, M.; Masson, S. J. Organomet. Chem. 2001, 617-618, 360.

(10) Poriel, C.; Ferrand, Y.; Le Maux, P.; Paul, C.; Rault-Berthelot, J.; Simonneaux, G. Chem. Commun. 2003, $18,2308$.

(11) Poriel, C.; Ferrand, Y.; Le Maux, P.; Paul-Roth, C.; Simonneaux, G.; Rault-Berthelot, J. J. Electroanal. Chem. 2005, 583, 92.

(12) Paul-Roth, C. O.; Simonneaux, G. Tetrahedron Lett. 2006, 47, 3275.

(13) Paul-Roth, C. O.; Simonneaux, G. C.R. Acad. Sci., Ser. IIb: Chim. 2006, 9, 1277.

(14) Paul-Roth, C.; Williams, G.; Letessier, J.; Simonneaux , G. Tetrahedron Lett. 2007, 48, 4317. 
(15) Ren, X.; Ren, A.; Feng, J.; Sun, C. Journal of Photochemistry and Photobiology A: Chemistry 2009, 203, 92.

(16) Drouet, S.; Paul-Roth, C.; Simonneaux, G. Tetrahedron 2009, 65, 2975.

(17) Drouet, S.; Paul-Roth, C. O. Tetrahedron 2009, 65, 10693.

(18) Drouet, S.; Merhi, A.; Argouarch, G.; Paul, F.; Mongin, O.; Blanchard-Desce, M.; Paul-Roth, C. O. Tetrahedron 2012, 68, 98.

(19) Baldo, M. A.; O'Brien, D. F.; You, Y.; Shoustikov, A.; Sibley, S.; Thompson, M. E.; Forrest, S. R. Nature 1998, 395, 151.

(20) Drouet, S.; Paul-Roth, C. O.; Fattori, V.; Cocchi, M.; Williams, J. A. G. New J. Chem. 2011, $35,438$.

(21) Mink, L. M.; Neitzel, M. L.; Bellomy, L. M.; Falvo, R. E.; Boggess, R. K.; Trainum, B. T.; Yeaman, P. Polyhedron 1997, 16, 2809.

(22) Pearson, C.; Cadd, D. H.; Petty, M. C.; Hua, Y. L. J. of Appl. Phys. 2009, 106, 064516.

(23) Ahn, J. H.; Wang, C.; Perepichka, I. F.; Bryce, M. R.; Petty, M. C. J. Mater. Chem. 2007, 17, 2996.

(24) Kalinowski, J.; Giro, G.; Cocchi, M.; Fattori, V.; Zamboni, R. Chem. Phys. Let. 2002, 277, 387.

(25) Drouet, S.; Ballut, S.; Rault-Berthelot, J.; Turban, P.; Paul-Roth, C. Thin Solid Films 2009, $517,5474$.

(26) Lee, W. A.; Grätzel, M.; Kalyanasundaram, K. Chem. Phys. Lett. 1984, 107, 308.

(27) Van Houten, J.; Watts, R. J. J. Am. Chem. Soc. 1976, 98, 4853. 